\title{
Superscaling in nuclei: A search for a scaling function beyond the relativistic Fermi gas model
}

\author{
A. N. Antonov, ${ }^{1,2}$ M. K. Gaidarov, ${ }^{1}$ D. N. Kadrev, ${ }^{1}$ M. V. Ivanov, ${ }^{1}$ E. Moya de Guerra, ${ }^{3}$ and J. M. Udias ${ }^{2}$ \\ ${ }^{1}$ Institute of Nuclear Research and Nuclear Energy, Bulgarian Academy of Sciences, Sofia 1784, Bulgaria \\ ${ }^{2}$ Departamento de Fisica Atomica, Molecular y Nuclear, Facultad de Ciencias Fisicas, Universidad Complutense de Madrid, \\ Madrid E-28040, Spain \\ ${ }^{3}$ Instituto de Estructura de la Materia, CSIC, Serrano 123, 28006 Madrid, Spain
}

(Received 20 February 2004; published 30 April 2004)

\begin{abstract}
We construct a scaling function $f\left(\psi^{\prime}\right)$ for inclusive electron scattering from nuclei within the coherent density fluctuation model (CDFM). The latter is a natural extension to finite nuclei of the relativistic Fermi gas model within which the scaling variable $\psi^{\prime}$ was introduced by Donnelly and collaborators. The calculations show that the high-momentum components of the nucleon momentum distribution in the CDFM and their similarity for different nuclei lead to quantitative description of the superscaling in nuclei. The results are in good agreement with the experimental data for different transfer momenta showing superscaling for negative values of $\psi^{\prime}$, including those smaller than -1 .
\end{abstract}

DOI: 10.1103/PhysRevC.69.044321

PACS number(s): 25.30.Fj, 21.60.-n, 21.10.Ft, 24.10.Jv

\section{INTRODUCTION}

The $y$ scaling in the inclusive scattering of high-energy electrons from nuclei has been actively studied in the last two decades (e.g., Refs. [1-5], and references therein) following the idea from Ref. [6]. It has been shown both theoretically and experimentally that a properly defined function (scaling function) depends only on a single variable $y$, the latter itself being a function of the transferred momentum $\mathbf{q}$ and energy $\omega[y=y(q, \omega)]$. It has been realized that at momenta $|\mathbf{q}|>500 \mathrm{MeV} / c$ and energies $\omega$ at or below the quasielastic peak position a nucleon is ejected in a "quasifree" way almost without the effects of the strong interaction. This scaling is usually called scaling of the first kind. It was shown that the scaling function is sensitive to the highmomentum components of the spectral function and nucleon momentum distribution. Thus its knowledge can provide important information about the dynamical ground-state properties, as well as about the reaction mechanism.

The comparison of the scaling functions of various nuclei with mass number $A \geqslant 4$ led to the conclusion that these functions are the same $[7,8]$. This behavior is called scaling of the second kind which, together with scaling of the first kind, leads to superscaling. These studies followed the theoretical concept of the superscaling introduced in Refs. $[9,10]$ considering the properties of the relativistic Fermi gas (RFG) model. The analyses of a large body of inclusive scattering data for nuclei from $A=4$ to $A=238$ in Refs. [7,8] demonstrated that the data in the low- $\omega$ side of the quasielastic peak exhibit superscaling behavior: the scaling functions are both independent on the momentum transfer and on the mass number. In these analyses the Fermi momentum for the RFG was used as a physical scale to define the proper scaling variable $\psi^{\prime}$ for each nucleus. An example of the superscaling behavior of the inclusive electron-scattering data for $q$ $\approx 1000 \mathrm{MeV} / \mathrm{c}$ and for the ${ }^{4} \mathrm{He},{ }^{12} \mathrm{C},{ }^{27} \mathrm{Al},{ }^{56} \mathrm{Fe}$, and ${ }^{197} \mathrm{Au}$ nuclei is given in Fig. 1 (the data are taken from Fig. 5 in Ref. [8]). An important conclusion has been drawn in Refs. $[7,8]$ that this universality is not restricted to the region of the quasielastic peak $\left(\left|\psi^{\prime}\right|<1\right)$ and that the superscaling extends to larger values of $\left|\psi^{\prime}\right|$ and thus, to the highmomentum components of the nucleon momentum distributions in nuclei. The existence of high-momentum components, and their similarity for different nuclei, is known to be due to the short-range and tensor nucleonnucleon correlations (see, e.g., Refs. [15,16], and references therein).

An extended study of scaling of the first and second kinds for inclusive electron scattering from nuclei with emphasis on the transverse response in the region above the quasielastic peak was performed in Ref. [11]. Approximate scaling of the second kind was observed and its modest breaking was supposed to be due to an inelastic version of the usual scaling variable. In Ref. [12] a unified relativistic approach used in the case of quasielastic kinematics was applied to the analysis of highly inelastic electron scattering. The complete inelastic spectrum was considered using the inelastic RFG model and its phenomenological extension based on direct

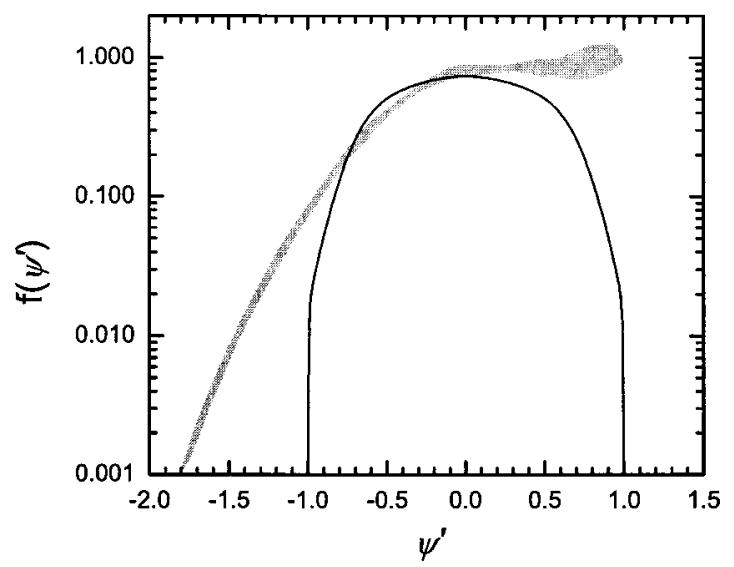

FIG. 1. Superscaling behavior of inclusive electron scattering. The gray area represents experimental data [8] for ${ }^{4} \mathrm{He},{ }^{12} \mathrm{C},{ }^{27} \mathrm{Al}$, and ${ }^{197} \mathrm{Au}$ at $q=1000 \mathrm{MeV} / c$. The solid line is the RFG scaling function calculated using Eq. (24) with $k_{F}=1.191 \mathrm{fm}^{-1}$ from Ref. [8]. 
fits to data, investigating the second-kind scaling behavior as well.

As emphasized in Ref. [18], the actual dynamical physics content of the phenomenon considered is more complex than that provided by the RFG framework. In particular, as noted there, the extension of the superscaling property to large negative values of $\psi^{\prime}\left(\psi^{\prime}<-1\right)$ is not predicted by the RFG model. This is seen in Fig. 1 where we also give a curve of the calculated RFG scaling function which is equal to zero for $\psi^{\prime} \leqslant-1$. Thus, it is worth considering the superscaling in theoretical approaches which go beyond the RFG model. One of them is the coherent density fluctuation model (CDFM) (e.g., Refs. [13-16]) which gives a natural extension of the Fermi-gas case to realistic finite nuclear systems. The main aim of the present work is to see to what extent superscaling can be explained using the CDFM. The theoretical scheme is given in Sec. II, while the results and the discussion are presented in Sec. III. The conclusions and the final remarks are given in Sec. IV.

\section{THE THEORETICAL SCHEME}

\section{A. Basic relationships of the CDFM}

The CDFM was suggested [13] and developed [14-17] as a model for studying characteristics of nuclear structure and nuclear reactions based on the local density and momentum distributions as basic variables of the theory and using the essential results of the infinite nuclear matter theory. The model is related to the $\delta$-function limit of the generator coordinate method (GCM) [18]. In the latter the total manyparticle wave function $\Psi\left(\left\{\mathbf{r}_{i}\right\}\right)$ of a system of $A$ nucleons is written in a form of a linear combination:

$$
\begin{aligned}
\Psi\left(\mathbf{r}_{1}, \ldots, \mathbf{r}_{A}\right)= & \int F\left(x_{1}, x_{2}, \ldots\right) \Phi\left(\mathbf{r}_{1}, \ldots, \mathbf{r}_{A} ; x_{1}, x_{2}, \ldots\right) \\
& \times d x_{1} d x_{2} \cdots,
\end{aligned}
$$

where the generating function $\Phi\left(\left\{\mathbf{r}_{i}\right\} ; x_{1}, x_{2}, \ldots\right)$ depends on the radius vectors of the nucleons $\left\{\mathbf{r}_{i}\right\}$ (spin and isospin variables are implied) and on the generator coordinates $x_{1}, x_{2}, \ldots$. The function $\Phi$ is usually chosen to be a Slater determinant built up from single-particle wave functions corresponding to a given construction potential parametrized by $x_{1}, x_{2}, \ldots$. The weight function $F\left(x_{1}, x_{2}, \ldots\right)$ can be determined using the variational principle as a solution of the Hill-Wheeler integral equation:

$$
\int\left[\mathcal{H}\left(x, x^{\prime}\right)-E \mathcal{I}\left(x, x^{\prime}\right)\right] F\left(x^{\prime}\right) d x^{\prime}=0
$$

In Eq. (2) the overlap kernel $\mathcal{I}\left(x, x^{\prime}\right)$ and the energy kernel $\mathcal{H}\left(x, x^{\prime}\right)$ have the following forms, respectively:

$$
\begin{gathered}
\mathcal{I}\left(x, x^{\prime}\right)=\left\langle\Phi\left(\left\{\mathbf{r}_{i}\right\}, x\right) \mid \Phi\left(\left\{\mathbf{r}_{i}\right\}, x^{\prime}\right)\right\rangle, \\
\mathcal{H}\left(x, x^{\prime}\right)=\left\langle\Phi\left(\left\{\mathbf{r}_{i}\right\}, x\right)|\hat{H}| \Phi\left(\left\{\mathbf{r}_{i}\right\}, x^{\prime}\right)\right\rangle,
\end{gathered}
$$

where $i=1,2, \ldots, A, \hat{H}$ is the Hamiltonian of the system and $x$ denotes a set of $x_{1}, x_{2}, \ldots$. For many-fermion systems the kernels $\mathcal{I}\left(x, x^{\prime}\right)$ and $\mathcal{H}\left(x, x^{\prime}\right)$ peak strongly at $x \sim x^{\prime}[19,20]$ and can be written in the form:

$$
\begin{aligned}
\mathcal{I}\left(x, x^{\prime}\right) & \simeq \mathcal{I}(x, x) \mathcal{G}\left(x-x^{\prime}\right), \\
\mathcal{H}\left(x, x^{\prime}\right) & \simeq \mathcal{H}(x, x) \mathcal{G}\left(x-x^{\prime}\right),
\end{aligned}
$$

where $\mathcal{G}$ is peaked at $x \sim x^{\prime}$. It was shown in Ref. [18] that the following $\delta$-function approximation for the kernels is valid in the GCM for the case of many-fermion systems

$$
\begin{gathered}
\mathcal{I}\left(x, x^{\prime}\right) \rightarrow \delta\left(x-x^{\prime}\right), \\
\mathcal{H}\left(x, x^{\prime}\right) \rightarrow-\frac{\hbar^{2}}{2 m_{e f f}} \delta^{\prime \prime}\left(x-x^{\prime}\right)+V\left(\frac{x+x^{\prime}}{2}\right) \delta\left(x-x^{\prime}\right),
\end{gathered}
$$

and that it leads to the Schrödinger-type of equation $[18,19,21]$ with an effective mass dependent on the generator coordinate (see also Ref. [16]).

In the following we use for simplicity only one generator coordinate. If the trial wave function $\Psi\left(\left\{\mathbf{r}_{i}\right\}\right)$ in Eq. (1) is normalized to the mass number $A$ and the weight function is determined under the condition

$$
\int_{0}^{\infty}|F(x)|^{2} d x=1
$$

then the $\delta$-function approximation (7) leads to the relationship:

$$
\int \Phi^{*}\left(\left\{\mathbf{r}_{i}\right\}, x^{\prime}\right) \Phi\left(\left\{\mathbf{r}_{i}\right\}, x\right) d \mathbf{r}_{1} \cdots d \mathbf{r}_{A}=A \delta\left(x-x^{\prime}\right) .
$$

Taking into account Eqs. (5) and (10) it was suggested in the CDFM that a $\delta$-function approximation leading to that of Eq. (10) holds in the case of many-fermion systems [14-16]:

$$
\begin{aligned}
& \int \Phi^{*}\left(\mathbf{r}, \mathbf{r}_{2}, \ldots, \mathbf{r}_{A}, x^{\prime}\right) \Phi\left(\mathbf{r}^{\prime}, \mathbf{r}_{2}, \ldots, \mathbf{r}_{A}, x\right) d \mathbf{r}_{2} \cdots d \mathbf{r}_{A} \\
& \cong \rho_{x, x}\left(\mathbf{r}, \mathbf{r}^{\prime}\right) \delta\left(x-x^{\prime}\right) .
\end{aligned}
$$

In Eq. (11) $\rho_{x, x}\left(\mathbf{r}, \mathbf{r}^{\prime}\right)$ is the one-body density matrix corresponding to the wave function $\Phi\left(\left\{\mathbf{r}_{i}\right\}, x\right)$ which can be formally written as

$$
\begin{aligned}
\rho_{x, x}\left(\mathbf{r}, \mathbf{r}^{\prime}\right) \equiv & \rho_{x}\left(\mathbf{r}, \mathbf{r}^{\prime}\right)=\frac{A}{\langle\Phi \mid \Phi\rangle} \int \Phi^{*}\left(\mathbf{r}, \mathbf{r}_{2}, \ldots, \mathbf{r}_{A} x\right) \\
& \times \Phi\left(\mathbf{r}^{\prime}, \mathbf{r}_{2}, \ldots, \mathbf{r}_{A}, x\right) d \mathbf{r}_{2} \cdots d \mathbf{r}_{A} .
\end{aligned}
$$

One can see that the integration of Eq. (11) (at $\mathbf{r}^{\prime}=\mathbf{r}$ ) over $\mathbf{r}$ using Eq. (12) leads to Eq. (10) which is the $\delta$-function limit for the overlap kernel in the GCM.

In the CDFM the generating function $\Phi\left(\left\{\mathbf{r}_{\mathbf{i}}\right\}, x\right)$ describes a system corresponding to a piece of nuclear matter with a one-body density matrix (ODM) of the form

$$
\rho_{x}\left(\mathbf{r}, \mathbf{r}^{\prime}\right)=3 \rho_{0}(x) \frac{j_{1}\left[k_{F}(x)\left|\mathbf{r}-\mathbf{r}^{\prime}\right|\right]}{\left[k_{F}(x)\left|\mathbf{r}-\mathbf{r}^{\prime}\right|\right]} \Theta\left(x-\frac{\left|\mathbf{r}+\mathbf{r}^{\prime}\right|}{2}\right)
$$

and uniform density 


$$
\rho_{x}(\mathbf{r})=\rho_{0}(x) \Theta(x-|\mathbf{r}|),
$$

where

$$
\rho_{0}(x)=\frac{3 A}{4 \pi x^{3}},
$$

and the generator coordinate $x$ is the radius of a sphere containing all $A$ nucleons in it.

In Eq. (13) $j_{1}$ is the first-order spherical Bessel function and

$$
\begin{aligned}
k_{F}(x) & =\left(\frac{3 \pi^{2}}{2} \rho_{0}(x)\right)^{1 / 3} \equiv \frac{\alpha}{x} \text { with } \quad \alpha=\left(\frac{9 \pi A}{8}\right)^{1 / 3} \\
& \simeq 1.52 A^{1 / 3}
\end{aligned}
$$

is the Fermi momentum of such a piece of nuclear matter. Using Eqs. (1) and (11) the ODM of the system in the CDFM can be obtained as a superposition of the ODM's from Eq. (13) [13-17]:

$$
\rho\left(\mathbf{r}, \mathbf{r}^{\prime}\right)=\int_{0}^{\infty} d x|F(x)|^{2} \rho_{x}\left(\mathbf{r}, \mathbf{r}^{\prime}\right) .
$$

The Wigner distribution function which corresponds to the ODM from Eq. (17) is

$$
W(\mathbf{r}, \mathbf{k})=\int_{0}^{\infty} d x|F(x)|^{2} W_{x}(\mathbf{r}, \mathbf{k}),
$$

where

$$
W_{x}(\mathbf{r}, \mathbf{k})=\frac{4}{(2 \pi)^{3}} \Theta(x-|\mathbf{r}|) \Theta\left(k_{F}(x)-|\mathbf{k}|\right) .
$$

Then the density $\rho(\mathbf{r})$ and the momentum distributions $n(\mathbf{k})$ in the CDFM are expressed by means of the same weight function $|F(x)|^{2}$ :

$$
\rho(\mathbf{r})=\int d \mathbf{k} W(\mathbf{r}, \mathbf{k})=\int_{0}^{\infty} d x|F(x)|^{2} \frac{3 A}{4 \pi x^{3}} \Theta(x-|\mathbf{r}|),
$$

and

$$
\begin{aligned}
n(\mathbf{k}) & =\int d \mathbf{r} W(\mathbf{r}, \mathbf{k})=\frac{4}{(2 \pi)^{3}} \int_{0}^{\infty} d x|F(x)|^{2} \frac{4 \pi x^{3}}{3} \Theta\left[k_{F}(x)-|\mathbf{k}|\right] \\
& =\frac{4}{(2 \pi)^{3}} \int_{0}^{\alpha / k} d x|F(x)|^{2} \frac{4}{3} \pi x^{3},
\end{aligned}
$$

both normalized to the mass number:

$$
\int \rho(\mathbf{r}) d \mathbf{r}=A, \quad \int n(\mathbf{k}) d \mathbf{k}=A .
$$

Different paths can be followed to find the function $F(x)$. Here, instead of solving the differential equation from the $\delta$-function approximation to the Hill-Wheeler integral equation (2), we adopt a convenient approach to the weight function $F(x)$ proposed in Refs. [13-16]. The function $F(x)$ is obtained by means of a known density distribution $\rho(r)$ for a given nucleus [from Eq. (20)]:

$$
|F(x)|^{2}=-\left.\frac{1}{\rho_{0}(x)} \frac{d \rho(r)}{d r}\right|_{r=x}, \quad \text { at } \quad d \rho(r) / d r \leqslant 0 .
$$

\section{B. The scaling function in the CDFM}

The scaling function in the RFG model expressed by the variable $\psi^{\prime}$ has the form [8]

$$
\begin{aligned}
f_{\mathrm{RFG}}\left(\psi^{\prime}\right)= & \frac{3}{4}\left(1-\psi^{\prime 2}\right) \Theta\left(1-\psi^{\prime 2}\right) \frac{1}{\eta_{F}^{2}}\left\{\eta_{F}^{2}+\psi^{\prime 2}\left[2+\eta_{F}^{2}\right.\right. \\
& \left.\left.-2 \sqrt{1+\eta_{F}^{2}}\right]\right\},
\end{aligned}
$$

where $\eta_{F}=k_{F} / m_{N}, m_{N}$ being the nucleon mass.

As shown in Ref. [8], the relationship between $\psi^{\prime}$ and the usual $y$ variable, in the approximation for the mass of the residual nucleus $M_{A-1}^{0} \rightarrow \infty$, is given by the expression

$$
\psi^{\prime}=\frac{y}{k_{F}}\left[1+\sqrt{1+\frac{1}{4 \kappa^{2}}} \frac{1}{2} \eta_{F}\left(\frac{y}{k_{F}}\right)+\mathcal{O}\left[\eta_{F}^{2}\right]\right],
$$

where $\kappa=q / 2 m_{N}$.

Our basic assumption within the CDFM is that the scaling function for a finite nucleus $f\left(\psi^{\prime}\right)$ can be defined by means of the weight function $|F(x)|^{2}$, weighting the scaling function for the RFG at given $x$ [i.e., for a given density $\rho_{0}(x)(15)$ and Fermi momentum (16)]. Thus the scaling function $f\left(\psi^{\prime}\right)$ in the CDFM will be an infinite superposition of the RFG scaling functions $f\left[\psi^{\prime}(x)\right]$.

Let us introduce the notation

$$
c \equiv \frac{1}{2 m_{N}} \sqrt{1+\frac{1}{4 \kappa^{2}}} .
$$

Then one can write from Eqs. (25) and (26), neglecting $\mathcal{O}\left[\eta_{F}^{2}\right]$, the scaling variable $\psi_{x}^{\prime}(y)$ corresponding to the relativistic Fermi gas with the density $\rho_{0}(x)$ (15) and Fermi momentum $k_{F}(x)(16)$ in the form

$$
\psi_{x}^{\prime}(y)=\frac{p(y)}{k_{F}(x)}=\frac{p(y) x}{\alpha},
$$

where for the cases of interest

$$
p(y)= \begin{cases}y(1+c y), & y \geqslant 0 \\ -|y|(1-c|y|), & y \leqslant 0, \quad|y| \leqslant 1 /(2 c) .\end{cases}
$$

For further use it is more convenient to introduce the notation

$$
\psi_{x}^{\prime}(y)=\frac{k_{F}}{k_{F}(x)} \frac{p(y)}{k_{F}}=\frac{k_{F}}{k_{F}(x)} \psi^{\prime}
$$

Using the $\Theta$ function in Eq. (24), the weighted scaling function for a finite nucleus can be presented by the integral 
TABLE I. Values of the parameters $R$ and $b$ (in $\mathrm{fm}$ ) used in the calculations and the results for $k_{F}\left(\mathrm{in} \mathrm{fm}^{-1}\right)$ obtained in the CDFM.

\begin{tabular}{lccc}
\hline \hline Nuclei & $R$ & $b$ & $k_{F}$ \\
\hline${ }^{4} \mathrm{He}$ & 1.710 & 0.290 & 1.201 \\
${ }^{12} \mathrm{C}$ & 2.470 & 0.420 & 1.200 \\
${ }^{27} \mathrm{Al} \mathrm{[23]}$ & 3.070 & 0.519 & 1.267 \\
${ }^{56} \mathrm{Fe} \mathrm{[23]}$ & 4.111 & 0.558 & 1.270 \\
${ }^{197} \mathrm{Au} \mathrm{[24]}$ & 6.419 & 0.449 & 1.335 \\
\hline \hline
\end{tabular}

$$
\begin{aligned}
f\left(\psi^{\prime}\right)= & \int_{0}^{\alpha /\left(k_{F}\left|\psi^{\prime}\right|\right)} d x|F(x)|^{2} \frac{3}{4}\left[1-\left(\frac{k_{F} x \psi^{\prime}}{\alpha}\right)^{2}\right] \\
& \times\left\{1+\left(\frac{x m_{N}}{\alpha}\right)^{2}\left(\frac{k_{F} x \psi^{\prime}}{\alpha}\right)^{2}\left[2+\left(\frac{\alpha}{x m_{N}}\right)^{2}\right.\right. \\
& \left.\left.-2 \sqrt{1+\left(\frac{\alpha}{x m_{N}}\right)^{2}}\right]\right\},
\end{aligned}
$$

where the momentum $k_{F}$ will not be a fitting parameter (as it is in the RFG model) for the different nuclei, but will be also calculated consistently in the CDFM,

$$
k_{F}=\int_{0}^{\infty} d x k_{F}(x)|F(x)|^{2}=\alpha \int_{0}^{\infty} d x \frac{1}{x}|F(x)|^{2}
$$

for each nucleus, with $\alpha$ given by Eq. (16). As can be seen from Eqs. (30), (29), and (28) in our approach the scaling function $f\left(\psi^{\prime}\right)$ is symmetric at the change of $\psi^{\prime}$ to $-\psi^{\prime}$ up to $\left|\psi^{\prime}\right|=\left(4 c k_{F}\right)^{-1}$.

The scaling function $f\left(\psi^{\prime}\right)$ has been calculated using Eq. (30) by means of the weight function $|F(x)|^{2}$ determined from its relationship to the density distribution $\rho(r)$ [Eq. (23)]. For the latter we used those obtained from experimental data on electron scattering from nuclei and muonic atoms.

\section{RESULTS OF CALCULATIONS AND DISCUSSION}

We calculated the scaling function $f\left(\psi^{\prime}\right)$ (30) for various nuclei and transfer momenta. A symmetrized diffused Fermi density distribution has been used for ${ }^{4} \mathrm{He}$ and ${ }^{12} \mathrm{C}$ [22] and a diffused Fermi distribution for the heavier nuclei. The values of the half-radius $R$ and diffuseness parameter $b$ are given in Table I together with the results for the CDFM Fermi momentum $k_{F}(31)$.

The results for the scaling function are compared with the experimental data from Refs. [7,8] which are given in our figures by a gray area. In Fig. 2 are presented the results for the scaling function in the CDFM for $q=1000 \mathrm{MeV} / c$ and for ${ }^{4} \mathrm{He},{ }^{12} \mathrm{C},{ }^{27} \mathrm{Al}$, and ${ }^{197} \mathrm{Au}$. The values of the parameters $R$ and $b$ for ${ }^{4} \mathrm{He}$ and ${ }^{12} \mathrm{C}$ (given in Table I) lead to charge rms radii $1.71 \mathrm{fm}$ and $2.47 \mathrm{fm}$, respectively, which coincide with the experimental ones [23]. The values of $R$ and $b$ for ${ }^{27} \mathrm{Al}$ are taken from Ref. [23]. The results of the CDFM scaling function (solid lines) are compared with the RFG predictions (dotted lines). In the RGF model, due to the $\Theta$ function in Eq. (24), $f\left(\psi^{\prime}\right)=0$ for $\psi^{\prime} \leqslant-1$. As can be seen, the CDFM

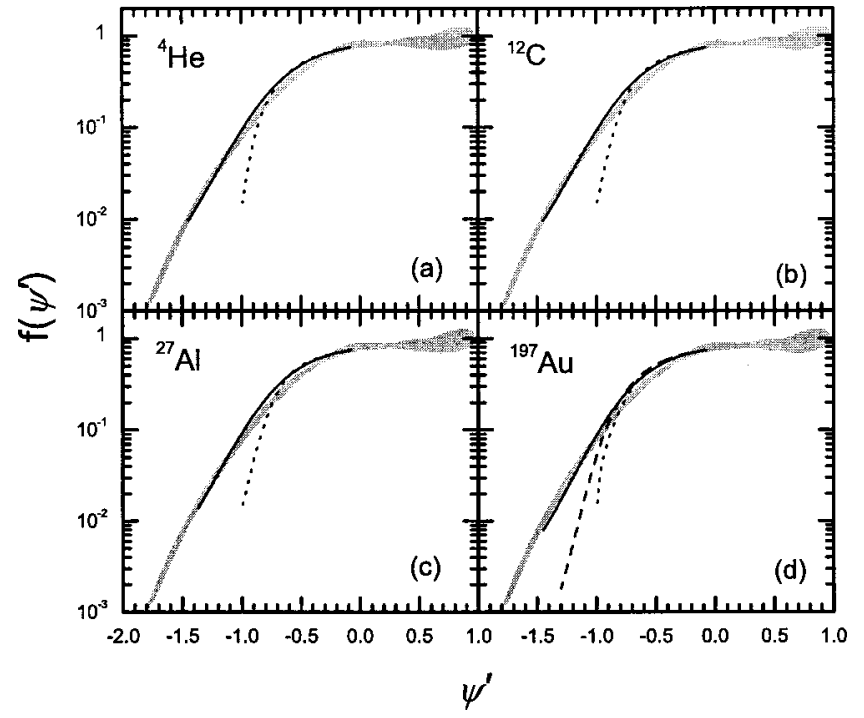

FIG. 2. Results for the scaling function in the CDFM (solid line) calculated using Eqs. (30) and (31) at $q=1000 \mathrm{MeV} / c$ and for ${ }^{4} \mathrm{He}$, ${ }^{12} \mathrm{C},{ }^{27} \mathrm{Al}$, and ${ }^{197} \mathrm{Au}$ (with $b=1.0 \mathrm{fm}$ for the latter) compared with the data (gray area) from Ref. [8]. The dotted line is the RFG result using Eq. (24). The dashed line in the case of ${ }^{197} \mathrm{Au}$ corresponds to the CDFM result with $b=0.449 \mathrm{fm}$.

results give a good agreement with the data for the interval for $\psi^{\prime}$ from 0 till $\psi^{\prime}<-1$ for all nuclei (including ${ }^{56} \mathrm{Fe}$ which is not shown). The only exception was observed for ${ }^{197} \mathrm{Au}$ using the values of the parameters $R=6.419 \mathrm{fm}$ and $b$ $=0.449 \mathrm{fm}$ given in Ref. [24], for which the result is shown in Fig. 2 by dashed line.

At this point we would like to note that, generally, the weight function $|F(x)|^{2}$ which is related to the density distribution [Eqs. (20) and (23)], is also related to the momentum distribution [Eq. (21)]. This connects through Eq. (30) the scaling function $f\left(\psi^{\prime}\right)$ with $n(k)$. The deviation of $f\left(\psi^{\prime}\right)$ from the data in the case of ${ }^{197} \mathrm{Au}(b=0.449 \mathrm{fm})$ is due to the particular $A$ dependence of $n(k)$ in the present approach [Eqs. (21) and (23)].

To understand the origin of the agreement for light and medium nuclei, and of the discrepancy for ${ }^{197} \mathrm{Au}$ with $b$ $=0.449 \mathrm{fm}$, we show in Fig. 3 the momentum distributions $n(k)$ (21) [Fig. 3(a)] and the weight functions $|F(x)|^{2}$ [Fig. 3(b)]. As seen in Fig. 3(a), for the ${ }^{12} \mathrm{C}$ and ${ }^{40} \mathrm{Ca}$ the CDFM momentum distributions depend weakly on $A$ and have similar high-momentum $\left(k>1 \mathrm{fm}^{-1}\right)$ tails. Inspection of Eqs. (21) and (30) [which involve the same weighting function $|F(x)|^{2}$ ] shows that the interval of interest for $\psi^{\prime}\left(-2 \leqslant \psi^{\prime}\right.$ $\leqslant 0$ ) corresponds to the $k$ interval $0 \leqslant k \leqslant-2.5 \mathrm{fm}^{-1}$ in $n(k)$. Thus the similar high-momentum tails of $n(k)$ in the light $\left({ }^{12} \mathrm{C}\right)$ and medium $\left({ }^{40} \mathrm{Ca}\right)$ nuclei lead to the similar CDFM scaling curves which are in agreement with the superscaling data.

The deviation between the CDFM scaling function and data in Fig. 2 for ${ }^{197} \mathrm{Au}(b=0.449 \mathrm{fm})$ takes place at $\psi^{\prime} \leqslant$ -1 and corresponds to values of the momentum $k \geqslant 3 \mathrm{fm}^{-1}$. The CDFM high-momentum tail of ${ }^{197} \mathrm{Au}$ for $b=0.449 \mathrm{fm}$ is much smaller than that of light and medium nuclei [see Fig. 3(a) causing the above mentioned deviation. In Fig. 3(b) we 

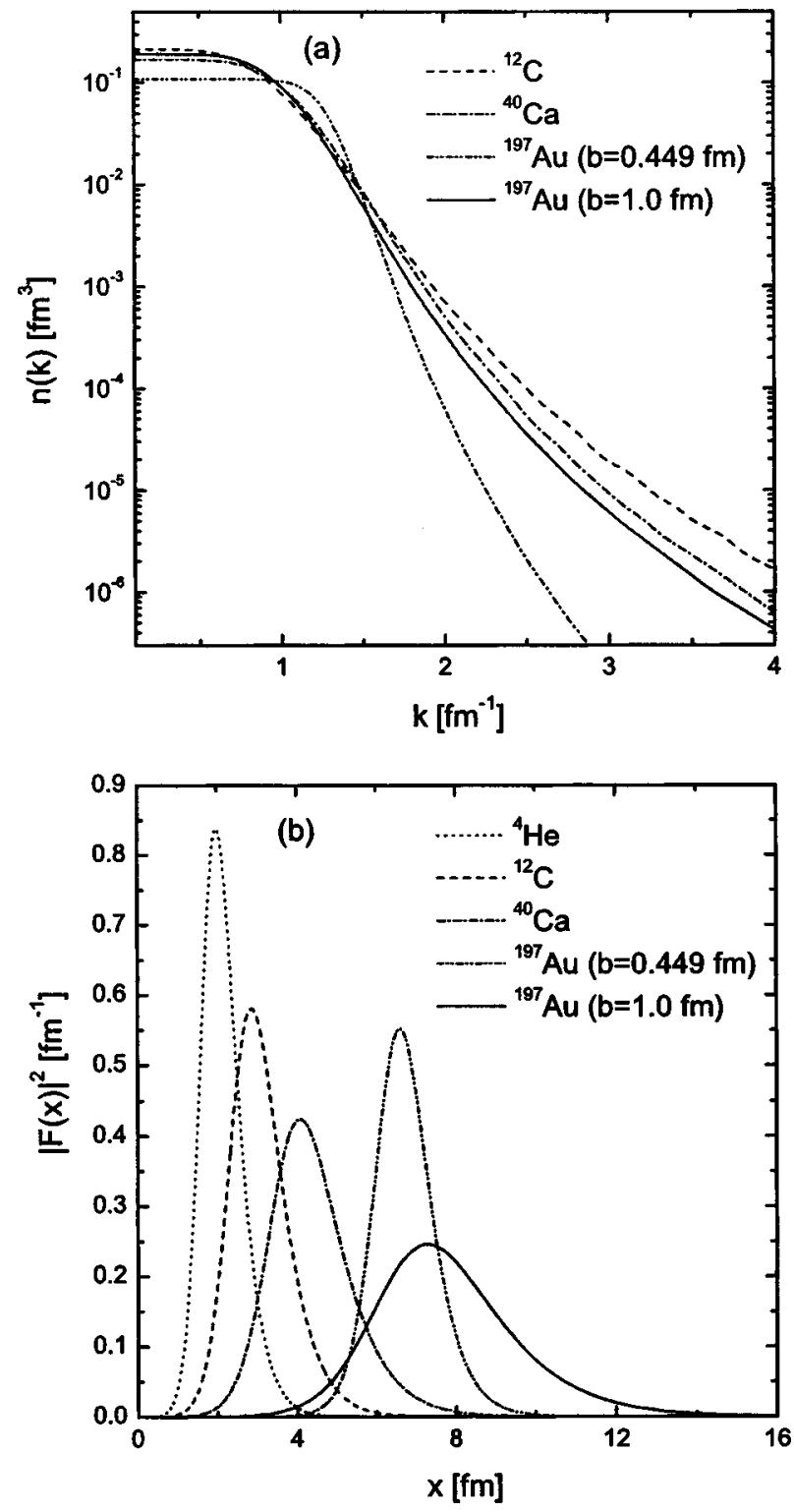

FIG. 3. (a) Nucleon momentum distribution $n(k)$ calculated in the CDFM using Eqs. (21) and (23) for ${ }^{12} \mathrm{C},{ }^{40} \mathrm{Ca}$, and ${ }^{197} \mathrm{Au}$ (for the latter with $b=0.449 \mathrm{fm}$ and $b=1.0 \mathrm{fm}$ ); (b) the weight function $|F(x)|^{2}$ of the CDFM calculated using Eq. (23) for ${ }^{4} \mathrm{He},{ }^{12} \mathrm{C},{ }^{40} \mathrm{Ca}$, and ${ }^{197} \mathrm{Au}$ (for the latter with $b=0.449 \mathrm{fm}$ and $b=1.0 \mathrm{fm}$ ).

give also the weight function $|F(x)|^{2}$ calculated in the CDFM for ${ }^{4} \mathrm{He},{ }^{12} \mathrm{C}$, ${ }^{40} \mathrm{Ca}$, and ${ }^{197} \mathrm{Au}$ using Eq. (23) and the corresponding density distributions mentioned above. As can be seen $|F(x)|^{2}$ is a one-peak function which for ${ }^{4} \mathrm{He},{ }^{12} \mathrm{C}$, and ${ }^{40} \mathrm{Ca}$ follows a particular trend with increasing $A$. The strength of the peak decreases (consequently its width increases) with increasing $A$, and the peak is displaced to higher $x$ values. This trend is broken by the behavior of $|F(x)|^{2}$ for ${ }^{197} \mathrm{Au}$ when we take $b=0.449 \mathrm{fm}$.

To improve the $A$ dependence of the momentum distribution in the CDFM for the heaviest nucleus, we use a procedure that may be somewhat artificial but which is useful to show the role of the obtained, more realistic, new highmomentum components of $n(k)$ on the scaling function. This can be achieved by taking an effective larger value of the parameter $b$ in the diffuse Fermi density distribution which is used to obtain the weight function $|F(x)|^{2}$ and, hence, the scaling function $f\left(\psi^{\prime}\right)$. We take the value $b=1.0 \mathrm{fm}$, for which the high-momentum components of $n(k)$ in ${ }^{197} \mathrm{Au}$ are similar to those in light and medium nuclei. This can be seen in Fig. 3(a) for ${ }^{197} \mathrm{Au}$ (solid line). The change of the behavior of the weight function $|F(x)|^{2}$ which leads to this shape of $n(k)$ for ${ }^{197} \mathrm{Au}$ can be seen in Fig. 3(b) (solid line). In this case the function $|F(x)|^{2}$ follows the trend previously observed for light and medium nuclei of decreasing strength (increasing width) with increasing $A$. The values of $|F(x)|^{2}$ for $0 \leqslant x \leqslant 5.2 \mathrm{fm}$ determine the behavior of $f\left(\psi^{\prime}\right)$ for $-1.5<\psi^{\prime}<-1$. As can be seen from Fig. 3(b) in this region $|F(x)|^{2}$ is quite different for $b=0.449 \mathrm{fm}$ and for $b=1.0 \mathrm{fm}$. The results of the calculations of the scaling function for ${ }^{197} \mathrm{Au}$ with $b=1.0 \mathrm{fm}$ are presented in Fig. 2 by a solid line and, as can be seen, they are in good agreement with the data. This confirms the view that the behavior of the scaling function is related to the properties of the momentum distribution at large values of $k\left(k>1.5 \mathrm{fm}^{-1}\right)$, and that the similarity of the high-momentum tails of $n(k)$ leads to the scaling of second kind.

Here we would like to note that the use of an effective value of $b$ for ${ }^{197} \mathrm{Au}$ can be merely seen as an artificial procedure to improve the $A$ dependence of $n(k)$ for the heaviest nucleus. This shows what would be the results of the CDFM for the scaling function when the high-momentum tails of $n(k)$ are realistic, even for the heaviest nucleus, and are similar to those of light and medium nuclei. We do not imply that the actual diffuseness of the density distribution of ${ }^{197} \mathrm{Au}$ should be that large. However, it is also worth pointing out that all the nucleons may contribute to the scaling function for the transverse electron scattering and that the diffuseness of the mass density for a nucleus like ${ }^{197} \mathrm{Au}$ may be larger than that of the charge density.

The results for the scaling function in the CDFM in the case of $q=1650 \mathrm{MeV} / c$ are given in Fig. 4 for ${ }^{4} \mathrm{He}$ and ${ }^{197} \mathrm{Au}$ [the latter with improved high-momentum tail of $n(k)$ ] and compared with the experimental data taken from Fig. 6 of Ref. [8]. The curves for ${ }^{12} \mathrm{C}$ and ${ }^{56} \mathrm{Fe}$ are not given since they are similar, in agreement with the data.

The result for the scaling function in the CDFM in the case of $q=500 \mathrm{MeV} / c$ for ${ }^{12} \mathrm{C}$ is given in Fig. 5. This result is also in good agreement with the experimental data for $q$ in the interval from 500 to $600 \mathrm{MeV} / \mathrm{c}$ given in Fig. 8 of Ref. [8].

One can see in Figs. 2 and 4 that the CDFM results tend to overestimate the data in the interval $-1 \leqslant \psi^{\prime} \leqslant-0.5$. We note that the origin of this is related to the predictions of the RFG model in the same region, as can be seen in Fig. 1.

\section{CONCLUSIONS AND FINAL REMARKS}

The results of the present work can be summarized as follows.

(i) We propose an extension of the RFG model to calculate the scaling function $f\left(\psi^{\prime}\right)$ in finite nuclei within the co- 


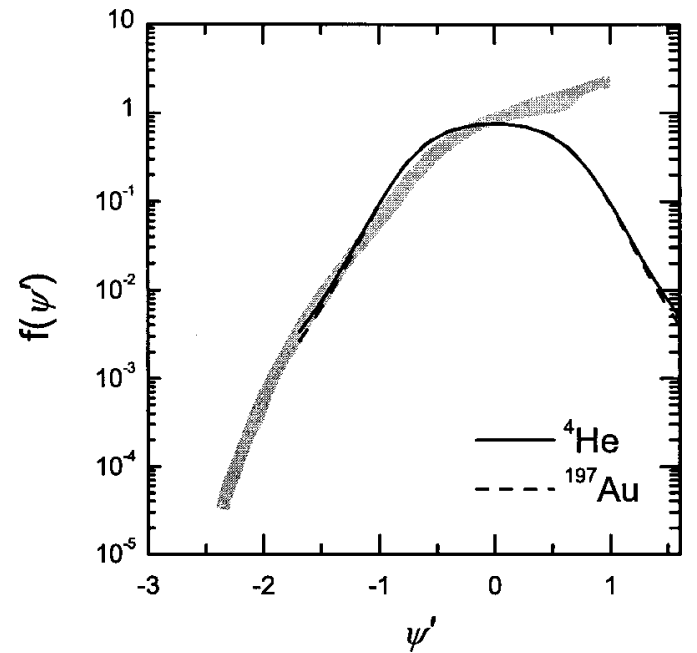

FIG. 4. Results of the CDFM for the superscaling functions of ${ }^{4} \mathrm{He}$ (solid line) and ${ }^{197} \mathrm{Au}$ (dashed line) at $q=1650 \mathrm{MeV} / c$ compared with the experimental data (gray area) from Ref. [8].

herent density fluctuation model (CDFM). In this model $f\left(\psi^{\prime}\right)$ is a weighted superposition of scaling functions for relativistic Fermi gases with different densities. The weight function is calculated using the known charge density distributions in nuclei.

(ii) We calculate the scaling function $f\left(\psi^{\prime}\right)$ for inclusive electron scattering for ${ }^{4} \mathrm{He},{ }^{12} \mathrm{C},{ }^{27} \mathrm{Al},{ }^{56} \mathrm{Fe}$, and ${ }^{197} \mathrm{Au}$ nuclei and for various values of the transfer momentum $|\mathbf{q}|=1650$, 1000 , and $500 \mathrm{MeV} / c$. The results agree with the available experimental data at different transferred momenta, and energies below the quasielastic peak position, showing superscaling for negative values of $\psi^{\prime}$ including also those smaller than -1 . This is an improvement over the RFG model predictions where the scaling function becomes abruptly zero beyond $\left|\psi^{\prime}\right|=-1$.

(iii) The sensitivity of the scaling function to the highmomentum components of the momentum distribution is analyzed in detail, especially so on the example of the ${ }^{197} \mathrm{Au}$ nucleus.

(iv) The scaling function obtained is symmetrical around $\psi^{\prime}=0$ up to $\left|\psi^{\prime}\right|=\left(4 c k_{F}\right)^{-1}$. It would also be interesting to search for models predicting an asymmetrical superscaling function $f\left(\psi^{\prime}\right)$ as the phenomenological one obtained by Ref. [25] and discussed in Ref. [12].

It is shown in our work that the superscaling in nuclei can be explained quantitatively on the basis of the similar behav-

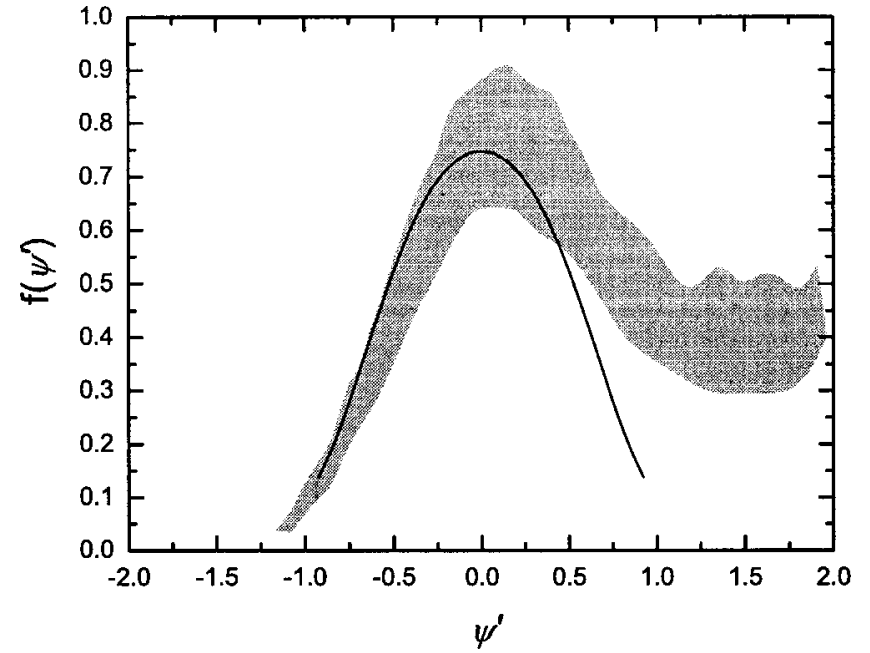

FIG. 5. Results of the CDFM for the superscaling function of ${ }^{12} \mathrm{C}$ at $q=500 \mathrm{MeV} / c$ (solid line) compared with the experimental data (gray area) for $q$ in the interval from 500 to $600 \mathrm{MeV} / c$ from Ref. [8].

ior of the high-momentum components of $n(k)$ in light, medium, and heavy nuclei which is known to be due to the short range and tensor correlations in nuclei. This suggests an alternative path for defining the weight function $F(x)$ within the generator coordinate method: a path in which $F(x)$ is built up from a phenomenological or a theoretical momentum distribution.

\section{ACKNOWLEDGMENTS}

One of the authors (A.N.A.) is grateful for warm hospitality to the Faculty of Physics of the Complutense University of Madrid and for support during his stay there to the State Secretariat of Education and Universities of Spain (N.Ref.SAB2001-0030). Four of the authors (A.N.A., M.K.G., D.N.K., and M.V.I.) are thankful to the Bulgarian National Science Foundation for partial support under the Contract No. Ф-905. This work was partly supported by funds provided by DGI of MCyT (Spain) under Contract Nos. BFM 2002-03562, BFM 2000-0600, and BFM 200304147-C02-01 and by the Agreement (2004 BG2004) between the CSIC (Spain) and the Bulgarian Academy of Sciences.
[1] D. Day, J. S. McCarthy, T. W. Donnelly, and I. Sick, Annu. Rev. Nucl. Part. Sci. 40, 357 (1990).

[2] I. Sick, D. Day, and J. S. McCarthy, Phys. Rev. Lett. 45, 871 (1980)

[3] E. Pace and G. Salme, Phys. Lett. 110B, 441 (1982).

[4] C. Ciofi degli Atti, E. Pace, and G. Salme, Phys. Lett. 127B, 303 (1983); Phys. Rev. C 43, 1155 (1991).

[5] C. Ciofi degli Atti and G. B. West, Phys. Lett. B 458, 447
(1999).

[6] G. B. West, Phys. Rep., Phys. Lett. 18C, 263 (1975).

[7] T. W. Donnelly and I. Sick, Phys. Rev. Lett. 82, 3212 (1999).

[8] T. W. Donnelly and I. Sick, Phys. Rev. C 60, 065502 (1999).

[9] W. M. Alberico, A. Molinari, T. W. Donnelly, E. L. Kronenberg, and J. W. Van Orden, Phys. Rev. C 38, 1801 (1988).

[10] M. B. Barbaro, R. Cenni, A. De Pace, T. W. Donnelly, and A. Molinari, Nucl. Phys. A643, 137 (1998). 
[11] C. Maieron, T. W. Donnelly, and I. Sick, Phys. Rev. C 65, 025502 (2002).

[12] M. B. Barbaro, J. A. Caballero, T. W. Donnelly, and C. Maieron, Phys. Rev. C 69, 035213 (2004).

[13] A. N. Antonov, V. A. Nikolaev, and I. Zh. Petkov, Bulg. J. Phys. 6, 151 (1979); Z. Phys. A 297, 257 (1980); 304, 239 (1982).

[14] A. N. Antonov, V. A. Nikolaev, and I. Zh. Petkov, Nuovo Cimento Soc. Ital. Fis., A 86A, 23 (1985).

[15] A. N. Antonov, P. E. Hodgson, and I. Zh. Petkov, Nucleon Momentum and Density Distributions in Nuclei (Clarendon Press, Oxford, 1988).

[16] A. N. Antonov, P. E. Hodgson, and I. Zh. Petkov, Nucleon Correlations in Nuclei (Springer-Verlag, Berlin, 1993).

[17] A. N. Antonov, Chr. V. Christov, E. N. Nikolov, I. Zh. Petkov, and P. E. Hodgson, Nuovo Cimento Soc. Ital. Fis., A 102A, 1701 (1989); A. N. Antonov, D. N. Kadrev, and P. E. Hodgson, Phys. Rev. C 50, 164 (1994).

[18] J. J. Griffin and J. A. Wheeler, Phys. Rev. 108, 311 (1957).

[19] K. Wildermuth and Y. C. Tang, A Unified Theory of the Nucleus (Vieweg, Braunschweig, 1977).

[20] W. Bauhoff, Ann. Phys. (N.Y.) 130, 307 (1980).

[21] P. A. M. Dirac, Proc. Cambridge Philos. Soc. 26, 376 (1930).

[22] V. V. Burov, D. N. Kadrev, V. K. Lukyanov, and Yu. S. Pol', Phys. At. Nucl. 61, 525 (1998).

[23] H. De Vries, C. W. De Jager, and C. De Vries, At. Data Nucl. Data Tables 36, 495 (1987).

[24] J. D. Patterson and R. J. Peterson, Nucl. Phys. A717, 235 (2003).

[25] J. Jourdan, Nucl. Phys. A603, 117 (1996). 